Original Article

\title{
BIODEGRADABLE LIPOSOMES FOR ACYCLOVIR-GOLD NANOPARTICLES AS AN EFFICIENT CARRIERFOR ENHANCED TOPICAL DELIVERY
}

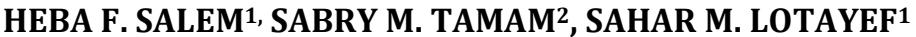

1Department of Pharmaceutics and Industrial Pharmacy, Faculty of Pharmacy, Beni-Suef University, Egypt, ${ }^{2}$ Department of Virology Faculty of Veterinary Medicine, Beni-Suef University, Beni-Suef, Egypt

Email: saharlotayef@gmail.com

Received: 22 Jan 2017 Revised and Accepted: 19 Jun 2017

\begin{abstract}
Objective: Gold nanoparticles and nanoliposomes are effective new technology in delivering the bioactive agents and enhancing their performance by increasing the bioavailability. The goal of the present study was the formulation of liposome for the use as a carrier for nanogold conjugated with acyclovir (ACV), an antiviral drug, to enhance ACV delivery.

Methods: The gold nanoparticles were used as a capping agent for ACV and sodium citrate was used as reducing agent for the gold. Transmission electron microscopy was used for characterization and to study the morphological and structural properties of drug-metallic nanostructures. Nanoliposomes were formulated using different molar ratios of a positive charge inducer (stearyl amine) or a negative charge inducer (diacetyl phosphate), lecithin, cholesterol and Span 60.

Results: Gold nanoparticles with a particle size of 10-20 nm were formed. This small size of the formed particles has a clear effect in reducing the gold nanoparticles toxicity and enhancing the cellular uptake. The amount of sodium citrate used in this preparation influences the size of the gold nanoparticle. The present study employed $1 \%$ trisodium citrate that contains a carboxylic group and this carboxylic group works as a reducing and capping agent to synthesize ACV-gold nanoparticles conjugate. Liposomal formula F6 had the highest entrapment efficiency approaching $42 \%$, the low particle size of $160 \mathrm{~nm}$, and zeta potential of $43.5 \mathrm{mV}$.
\end{abstract}

Conclusion: It is evident from the study that the liposomes can be used as a carrier of ACV conjugated with gold nanoparticles. This new strategy could be used successfully in the treatment of viral infection.

Keywords: Acyclovir, Gold Nanoparticles, Liposome, Dicetyl phosphate, Stearylamine

(c) 2017 The Authors. Published by Innovare Academic Sciences Pvt Ltd. This is an open access article under the CC BY license (http://creativecommons.org/licenses/by/4.0/) DOI: http://dx.doi.org/10.22159/ijpps.2017v9i8.17243

\section{INTRODUCTION}

Acyclovir (ACV) is an antiviral drug that is primarily used for the treatment of herpes simplex virus infections, chickenpox, and prevention of cytomegalovirus infections following transplant and infections due to Epstein-Barr virus [1]. ACV is available by mouth and intravenously [2]. ACV is also categorised class III drug because of its high solubility and low permeability according to the Biopharmaceutical Classification System (BCS) [3]. The intrinsic solubility of ACV was $1.2 \mathrm{mg} / \mathrm{ml}$ as measured by the acid-base titration method. ACV has dissociation constant (pka) of 2.34 and 9.23 and partition coefficient (P-Octanol) of 0.023 . This low partition coefficient may reflect the low membrane permeability of ACV [4].

In human, ACV showed poor and variable oral bioavailability (15\%$30 \%$ ), probably due to the relatively low lipophilicity of the drug so the absorption rate limiting step is its poor permeability. Also, systemic administration of ACV may cause mild to severe side effects for example nephrotoxicity so the topical application of ACV is desirable [5]. The epidermal application of ACV is an effective treatment for localised herpetic lesions [6].

Nanoparticles can enhance the properties of drugs such as size, distribution and morphology [7]. Liposomes have been proposed as delivery systems for topical application. Unlike many parts of the body, the drug delivery to the tissue via the skin is relatively easy [8]. The treatment of many skin diseases is difficult mainly when the drug penetration into deeper skin strata is required [9]. The skin permeability coefficient can be increased by using many skin penetration enhancers and this leads to increase in the drug levels within deep skin strata, i.e. deeper stratum corneum, epidermis and dermis [10]. Thus, liposomes can be used as transdermal drug delivery systems $[8,11]$.
In this study, we present the synthesis and characterization of gold nanoparticles conjugated with ACV-loaded liposome to be used for successful antiviral topical therapy [12]. The method of preparation of gold nanoparticles was pioneered by J. Turkevich et al. in 1951 and was refined by G. Frens in the 1970. In general, this method was used to produce modestly monodisperse spherical gold nanoparticles suspended in water of around $10-20 \mathrm{~nm}$ in diameter. This technique involves the reaction of small amounts of hot chloroauric acid with small amounts of sodium citrate solution. The colloidal gold will be formed because the citrate ions works as a capping an reducing agent [13].

\section{MATERIALS AND METHODS}

\section{Materials}

Acyclovir (ACV), egg lecithin, cholesterol, stearyl amine, diacetyl phosphate and Span 60 were purchased from Sigma-Aldrich (Egypt). Hydrogen tetrachlorocuprate and trisodium citrate dehydrate were obtained from El Gomhoria CO. (Egypt). Disodium hydrogen orthophosphate (Na2HPO4), potassium dihydrogen orthophosphate (KH2PO, ethanol $(99 \% \mathrm{v} / \mathrm{v})$, methanol and chloroform were purchased from Fisher Scientific (Egypt). All reagents and chemicals were of analytical grade. Phosphate buffer solution (PBS, pH 5.5) was formed using potassium dihydrogen orthophosphate and disodium hydrogen orthophosphate.

Preparation of acyclovir-gold nanoparticle conjugates (AGC)

Gold nanoparticles were prepared according to the method of Turkevich-Frens [14] with some modification. Briefly, $20 \mathrm{ml}$ of 1.0 mmol HAuCl4 was added to a $50 \mathrm{ml}$ Erlenmeyer flask on a stirring hot plate then add a magnetic stir bar and bring the solution to a rolling boil. Then quickly add $2 \mathrm{ml}$ of a $1 \%$ solution of trisodium citrate dihydrate to the rapidly stirred boiling solution. The gold 
nanoparticle gradually formed as the citrate reduces the gold (III). After the solution has turned deep red or $10 \mathrm{~min}$ has elapsed, gold nanoparticles were allowed to rest at room temperature for $24 \mathrm{~h}$. The resulting solution was twice centrifuged at 15,000 rpm for $45 \mathrm{~min}$, washed with double distilled water, and finally redispersed in distilled water. The gold nanoparticles were functionalized with ACV in an ice bath and vigorous mixing conditions at a molar ratio of 7:100. A solution of ACV was added drop by drop to the solution of the gold nanoparticles and then mixed for $1 \mathrm{~h}$ then centrifuged three times at 15,000 rpm for 1 $\mathrm{h}$ and washed with PBS and finally redispersed in PBS.

\section{Preparation of the Nanoliposomes}

The liposomes were formed using the thin film hydration technique [15]. Charged liposomes positively or negatively were obtained using phosphatidylcholine (PC), cholesterol (Ch.) and span 60, in combination with stearylamine or diacetyl phosphate. Phosphatidylcholine, cholesterol and span 60 were dissolved in a mixture of ethanol $(99 \% \mathrm{v} / \mathrm{v})$ : chloroform at a ratio of one to three (table 1). The organic solvents were vaporized under vacuum conditions within a rotary evaporator at a temperature of $40^{\circ} \mathrm{C}$ for $25 \mathrm{~min}$. Gold nanoparticles functionalized with ACV in PBS (10 ml, pH 5.5) were added to hydrate the prepared film. The preparations were sonicated for a period of $2 \mathrm{~h}$.

After that, the liposomal dispersion was subjected to centrifugation using a refrigerated centrifuge at $21,000 \mathrm{rpm}$ for $2 \mathrm{~h}$ at a temperature of $15{ }^{\circ} \mathrm{C}$ (High-Speed Refrigerated Centrifuge, CR22N; Hitachi Ltd., Tokyo, Japan). Following the centrifugation, the supernatant was carefully siphoned off to separate the free acyclovir-gold nanoparticles. The liposomes were in the sediment and contained the entrapped drugs. The sediment was suspended in $5 \mathrm{ml}$ of phosphate buffer (pH 5.5) in order to be evaluated. The liposomal dispersion (contain no free gold nanoparticles) was stored at a constant temperature of $4{ }^{\circ} \mathrm{C}$ within glass vials.

Table 1: Composition of both positively and negatively charged liposomal formulations in molar ratio

\begin{tabular}{llllll}
\hline Liposomes & Phosphatidylcholine $(\mathbf{w} / \mathbf{v})$ & Cholesterol $(\mathbf{w} / \mathbf{v})$ & Span $\mathbf{6 0}(\mathbf{w} / \mathbf{v})$ & Stearylamine $(\mathbf{w} / \mathbf{v})$ & Dicetyl phosphate $(\mathbf{w} / \mathbf{v})$ \\
\hline F1 & 3 & 1 & 0.10 & 0 & 0 \\
F2 & 2 & 1 & 0.10 & 0 & 0 \\
F3 & 1 & 1 & 0.10 & 0 & 0 \\
F4 & 1 & 1 & 0.10 & 0.06 & 0 \\
F5 & 1 & 1 & 0.10 & 0.12 & 0 \\
F6 & 1 & 1 & 0.10 & 0.18 & 0.06 \\
F7 & 1 & 1 & 0.10 & 0.12 \\
F8 & 1 & 1 & 0.10 & 0 & 0.18 \\
F9 & 1 & 1 & 0.10 & 0 & \\
\hline
\end{tabular}

Abbreviations: F1, PC: Ch: Span 60 at a molar ratio of 3:1:1; F2, PC: Ch: Span 60 at a molar ratio of 2:1:1; F3, PC: Ch: Span 60 at a molar ratio of 1:1:1; F4, PC: Ch: Span 60:SA at a molar ratio of 1:1:1:0.06; F5, PC: Ch: Span 60:SA at a molar ratio of 1:1:1:0.12; F6, PC: Ch: Span 60:SA at a molar ratio of 1:1:1:0.18; F7, PC: Ch: Span 60:DCP at a molar ratio of 1:1:1:0.06; F8, PC: Ch: Span 60:DCP at a molar ratio of 1:1:1:0.12; F9, PC: Ch: Span 60:DCP at a molar ratio of 1:1:1:0.18; Ch, cholesterol; DCP, dicetyl phosphate; PC, phosphatidylcholine; SA, stearylamine.

\section{Characterization of the prepared liposomes}

\section{Determination of liposomes entrapment efficiency}

A volume of $(5 \mathrm{ml})$ of the liposome preparation was centrifuged at $21,000 \mathrm{rpm}$ at $15{ }^{\circ} \mathrm{C}$ for $120 \mathrm{~min}$. The sediment was redispersed in 5 $\mathrm{ml}$ of PBS. The centrifugation was repeated two times. The supernatant was separated from the liposomes. In a $10 \mathrm{ml}$ volumetric flask, $1 \mathrm{ml}$ of the prepared liposomes were diluted to a volume of $5 \mathrm{ml}$ with methyl alcohol. The amount of the encapsulated ACV-gold nanoparticles was determined by the atomic absorption spectrometer (A Analyst 200; PerkinElmer Inc., Waltham, MA, USA) at $243 \mathrm{~nm}$, where the samples were in an oil bath for $1 \mathrm{~h}$ processed at $120{ }^{\circ} \mathrm{C}$ in diluted aqua regia. The percentage of encapsulation efficiency of gold nanoparticles was calculated using the equation:

$$
\left.\% \mathrm{EE}=\frac{\text { TD-UED }}{\text { TD }} \times 100--------1\right)
$$

Where EE \% is the percent of the encapsulation efficiency, TD is the total gold nanoparticles amount, and UED is the un-encapsulated gold nanoparticles amount.

\section{Particle size analysis and zeta potential measurements}

The prepared liposomes, the colloidal ACV-gold nanoparticles and gold nanoparticles were characterized for their sizes and polydispersity indices by the Malvern PCS4700 instrument (Malvern Instruments, Malvern, UK). The formulations of liposomes were diluted with HEPES (4-(2-hydroxyethyl)-1-piperazineethanesulfonic acid) buffer (10 mmol, $\mathrm{pH} 5.5)$ to reduce the turbidity of the dispersions. The zeta potential of the prepared liposomes, the solution of colloidal acyclovir-gold nanoparticles and gold nanoparticles were measured by a Zetasizer Nano-ZS, Model ZEN 3600 (Malvern Instruments).

\section{Transmission electron microscope (TEM)}

TEM was used to investigate and characterize the prepared colloidal gold nanoparticles and ACV-gold nanoparticles to show their size, morphology, and shape. The prepared colloidal gold nanoparticles were processed by mounting each one under investigation onto copper grids in order to absorb the nanoparticles from the suspension. The samples were stained with $2.5 \%$ uranyl acetate for $30 \mathrm{~s}$ and then dried. The specimens were observed under a TEM (TEM-1010 T; JEOL, Tokyo, Japan) operated at $120 \mathrm{kV}$.

\section{In vitro release study of the liposomes}

The in vitro ACV-gold nanoparticle release of the liposomal formulations was estimated using dialysis tubing (molecular weight cutoff of $40 \mathrm{k} \mathrm{Da}$ ). In $25 \mathrm{ml}$ of phosphate buffer (pH 5.5) the dialysis bags were suspended and placed within the dissolution flask of the dissolution apparatus with a constant temperature of $37^{\circ} \mathrm{C} \pm 0.5^{\circ} \mathrm{C}$. At predetermined time intervals for a period of $12 \mathrm{~h}$, samples of $1 \mathrm{ml}$ were withdrawn from the dissolution medium and then diluted to 1:10 in distilled water. To maintain the sink condition the dissolution medium was replaced with the same volumes of a fresh dissolution medium of phosphate buffer ( $\mathrm{pH}$ 5.5). An atomic absorption spectrometer was used in measuring the release of gold nanoparticles (An Analyst 200; PerkinElmer Inc.) at $243 \mathrm{~nm}$. The release of gold nanoparticles was determined using the following equation:

$$
\left.\% \text { Drug release }=\frac{Q t}{Q_{i}} \times 100----------2\right)
$$

Where, $Q_{i}$ and $Q_{t}$ are the initial amounts of gold nanoparticles entrapped in the liposomes, and the released amount of gold nanopartat time t. All experimental procedures were repeated three times.

\section{RESULTS AND DISCUSSION} Preparation and characterization of ACV-gold nanoparticles
conjugates

Gold nanoparticles were synthesised by reduction of tetrachlorocuprate by using trisodium citrate that works as reducing agent and capping agent. The size of gold nanoparticles was $16 \mathrm{~nm}$. The color changed from pale yellow to wine red upon formation of 
the gold nanoparticles. Investigation of the gold nanoparticle using TEM indicates that nanoparticles were spherical and homogeneous as shown in fig. 1(a). Various ACV: gold nanoparticle ratios (4:100 to 9:100) were formed. ACV conjugation with the gold nanoparticles reached almost $100 \%$ at ratios 7:100, where no free ACV using UVvisible spectroscopy at $252 \mathrm{~nm}$ and the color changed from wine red to purple. Above this ratio blue precipitate is formed and free ACV was present. The formation of ACV conjugated gold nanoparticles was confirmed using TEM where particle size increased to $20 \mathrm{~nm}$ as shown in Figure1 (b). ACV: gold nanoparticle ratio of 7:100 was selected to be used in the subsequent studies.

\section{Encapsulation efficiency}

The encapsulation efficiency of the drug into liposome was expressed as a loading efficiency percent. The percent fraction of the overall input drug entrapped in the liposomes is known as loading efficiency. Loading efficiency was calculated using the following equation:

$$
\left.\% \mathrm{EE}=\frac{\mathrm{TD}-\mathrm{UED}}{\mathrm{TD}} \times 100--------3\right)
$$

Where EE\%: percentage of encapsulation, TD: total drug amount, UED: the amount of free drug.

Our findings in table 2 show that ACV-gold nanoparticles have been successfully encapsulated into liposomes. Entrapment efficiency of gold nanoparticles ranged from $25.74 \pm 3.01$ to $41.58 \pm 2.49$. The increase of cholesterol percent results in increasing the encapsulation percentage of ACV-gold nanoparticles and increasing the rigidity of the lipid bilayer of liposomes [16].

The charge inducers effect on the entrapment efficiency of ACV-gold nanoparticles in liposomes was obvious with formulations F4-F9. The positively charged liposomes, which contained PC/Ch/Span 60/SA in a molar ratio of 1: 1: 0.1: 0.18 , showed the highest EE \%, and the liposomal formulation (F3) formed from PC/Ch/Span 60 in a molar ratio of 1:1:0.1 exhibited entrapping efficiency more than the liposomal formulations F7, F8, and F9.

Table 2: Entrapment efficiency of the prepared neutral and charged liposomal formulations

\begin{tabular}{ll}
\hline Formulation & $\begin{array}{l}\text { Entrapment efficiency of loaded ACV-gold } \\
\text { nanoparticles }\end{array}$ \\
\hline F1 & $30.06 \pm 1.71$ \\
F2 & $33.22 \pm 2.48$ \\
F3 & $36.20 \pm 1.08$ \\
F4 & $35.45 \pm 1.99$ \\
F5 & $37.18 \pm 2.27$ \\
F6 & $41.58 \pm 2.49$ \\
F7 & $32.47 \pm 1.50$ \\
F8 & $30.88 \pm 1.97$ \\
F9 & $25.74 \pm 3.01$ \\
\hline
\end{tabular}

Abbreviations: F1, PC: Ch: Span 60 at a molar ratio of 3:1:1; F2, PC: Ch: Span 60 at a molar ratio of 2:1:1; p3, PC: Ch: Span 60 at a molar ratio of 1:1:1; F4, PC: Ch: Span 60:SA at a molar ratio of 1:1:1:0.06; F5, PC: Ch: Span 60:SA at a molar ratio of 1:1:1:0.12; F6, PC: Ch: Span 60:SA at a molar ratio of 1:1:1:0.18; F7, PC: Ch: Span 60:DCP at a molar ratio of 1:1:1:0.06; F8, PC: Ch: Span 60:DCP at a molar ratio of 1:1:1:0.12; F9, PC:
Ch: Span 60:DCP at a molar ratio of 1:1:1:0.18; Ch, cholesterol; DCP, diacetyl phosphate; $\mathrm{PC}$, phosphatidylcholine; SA, stearyl amine

Where $(n=3 \pm S D)$

These orders of EE \% may be due to gold nanoparticles have a negative charge, so an electrostatic attraction is formed between the drug and the positive charge inducer stearyl amine. An increase in the EE \% when compared with the negative charged liposome is due to this attraction force. On the contrary, an electrostatic repulsion is formed between the drug and the negative charge inducer, diacetyl phosphate, which leads to a decrease in the EE \% of less than those for the neutral formulations. There were Similar results during the addition of indomethacin as a charge inducer into liposomes and acetazolamide liposomes $[17,18]$. Consequently, the encapsulation efficiency of the formulation F6 is the highest. One-way ANOVA shows a significant difference between all pairs at $P<0.05$.

Particle size analysis, zeta potential measurements, and morphology

The results of particle size measurements for liposomes are shown in table 3. The mean particle size was to be $122.1 \pm 10.1 \mathrm{~nm}$ for neutral liposomal formulation F3. Formulations F4-F6 showed mean particle diameters of $124.9 \pm 5.5 \mathrm{~nm}, 155.0 \pm 4.2 \mathrm{~nm}$ and $159.1 \pm 12.0$ $\mathrm{nm}$, respectively, which were higher than those of liposomal formulations F1-F3. Formulations F7-F9 showed a mean particle size of $135.5 \pm 14.1 \mathrm{~nm}, 149.9 \pm 4.9 \mathrm{~nm}$, and $152.1 \pm 8.9 \mathrm{~nm}$, respectively, which was higher than that of liposomal formulations F1-F3. These results explained on the basis of increasing the spacing between the adjacent bilayers of liposomes as a result of the inclusion of a charge inducer within the liposomes, which, results in the formation of larger one compared with neutral liposomes [19]. The encapsulation efficiency results of gold nanoparticle-loaded liposomes can be explained on the same basis, the gold nanoparticles have the negative charge. Consequently, the liposomal formulation F6 has the highest encapsulation efficiency. Moreover, the sample of the prepared solution of colloidal ACV-gold nanoparticles had a particle size of $20.3 \pm 4.0 \mathrm{~nm}$. The liposomal preparations had a closed bilayer structure and the size ranging from $80-120 \mathrm{~nm}$. Those findings were consistent with the results obtained from the particle size measurements, as shown in table 3 .

\section{ACV, acyclovir}

The liposome F9 has the highest zeta potential, and the lowest value is produced with the liposomes F6. The results indicate that all preparations are stable according to reported literature [20]. The particles which have low zeta potential, no force will prevent them from the flocculating. The difference between stable and unstable suspension preparation is considered at either $+30 \mathrm{mV}$ or $-30 \mathrm{mV}$. A stable condition for colloidal preparation is considered when the zeta potential of the prepared suspension is more than $+30 \mathrm{mV}$ or its negativity is more than $-30 \mathrm{mV}$ [21]. The measurement of zeta potential is significant for characterization of gold nanoparticles. The zeta potential of the prepared solution of colloidal ACV-gold nanoparticles was $-29.75 \pm 2.45 \mathrm{mV}$. This negative charge of gold nanoparticles is high so it is considered a basic indicator for their particle size. The negative zeta potential charge of nanoparticles indicates that the particle size is less than $100 \mathrm{~nm}$ [22].

Table 3: Particle size, polydispersity index, and zeta potential of the prepared formulations

\begin{tabular}{llll}
\hline Formulation & Particle size $(\mathbf{n m})$ & Polydispersity index & Zeta potential (mV) \\
\hline F1 & $87.1 \pm 10.6$ & 0.238 & $-47.4 \pm 2.01$ \\
F2 & $109.5 \pm 9.1$ & 0.291 & $-53.8 \pm 1.82$ \\
F3 & $122.1 \pm 10.1$ & 0.205 & $-56.4 \pm 1.41$ \\
F4 & $124.9 \pm 5.5$ & 0.308 & $24.1 \pm 1.54$ \\
F5 & $155.0 \pm 4.2$ & 0.241 & $29.4 \pm 1.76$ \\
F6 & $159.1 \pm 12.0$ & 0.441 & $43.5 \pm 2.12$ \\
F7 & $135.5 \pm 14.1$ & 0.346 & $-57.9 \pm 2.25$ \\
F8 & $149.9 \pm 4.9$ & 0.259 & $-58.1 \pm 1.70$ \\
F9 & $152.1 \pm 8.9$ & 0.214 & $-64.0 \pm 0.59$ \\
Gold nanoparticles & $16.3 \pm 4.0$ & 0.189 & $-40.75 \pm 2.45$ \\
ACV-gold nanoparticles & $20.3 \pm 4.0$ & 0.190 & $-29.75 \pm 2.45$ \\
\hline
\end{tabular}

Where $(\mathrm{n}=3 \pm \mathrm{SD}), \mathrm{ACV}$, Acyclovir 


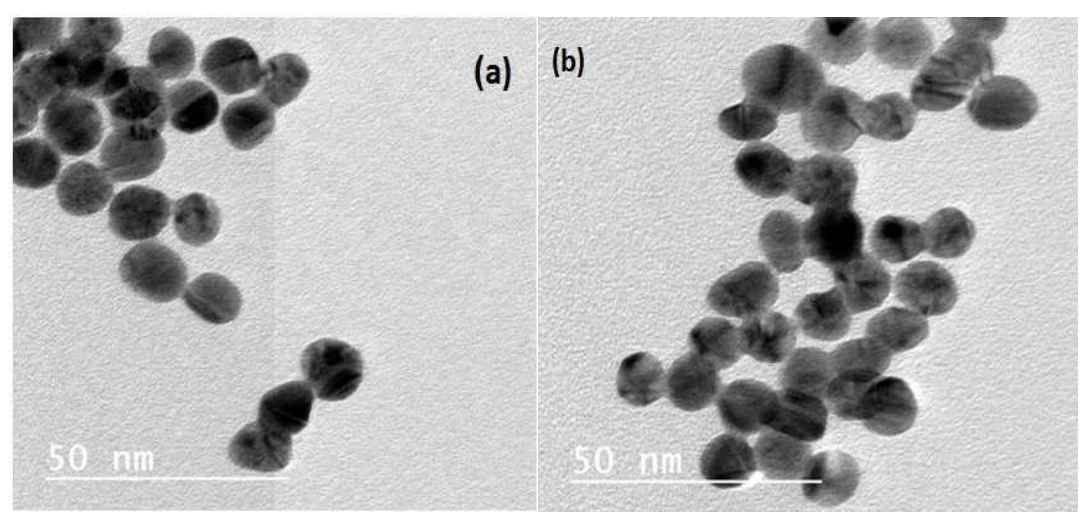

Fig. 1: TEM of gold nanoparticles: (a) gold nanoparticles, (b) ACV-gold nanoparticles

Liposomal preparations F1-F3 carry a strong negative charge as shown in table 3 . These results may be due the low ionic strength and the composition of the liposome. The nonionic surfactant increased the negative charge of the preparation. These results are in agreement with that have been found by sureewan et al. [23]. Moreover, gold nanoparticles may help in the high negative charge of gold nanoparticle-loaded liposomes, where the zeta potential of ACV-gold nanoparticles was $-29.74 \pm 2.45 \mathrm{mV}$. The ionic strength of liposomal dispersion may affect the zeta potential of the prepared liposomes. Crommelin [24] has reported that the liposomal zeta potential was larger at low ionic strength.

\section{In vitro drug release}

The release of ACV-gold nanoparticles from the prepared nanoliposomes within a phosphate-buffered saline medium of $\mathrm{pH}$ 5.5 was investigated. The percentage of cumulative release of ACVgold nanoparticles from the liposomes is presented in fig. (2). From cumulative release profiles fig. (2), it is apparent that the liposomes F6 showed the slowest rate of ACV-gold nanoparticles release. In contrast, the liposomes F9 showed the fastest release rate of ACVgold nanoparticles release. The gold nanoparticle concentration was measured using atomic absorption spectrophotometry. ACV-Gold nanoparticles have a strong negative charge so; the electrostatic repulsion which occurs between the negatively charged liposomes and ACV-gold nanoparticles may be the cause for the elevated release rate of gold nanoparticles. The findings obtained was in agreement with those obtained by hosny [25] who found that the electrostatic repulsion between the negatively charged liposomes and ciprofloxacin can increase the rate of drug release. Linear regression analysis of the release data fitted into zero-order.

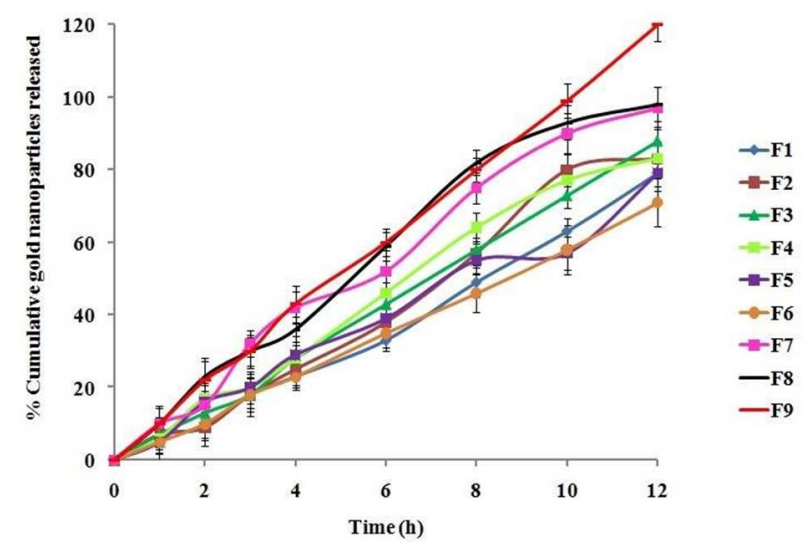

Fig. 2: The effect of varying molar ratios of the positive charge inducer (stearyl amine) and the negative charge inducer (dicetyl phosphate) on the cumulative release profile of gold nanoparticles from the prepared positive liposomes (F4, F5, and F6) and negative liposomes (F7, F8 and F9) compared with the liposomes (F1, F2 and F3), Where ( $=3 \pm S D)$

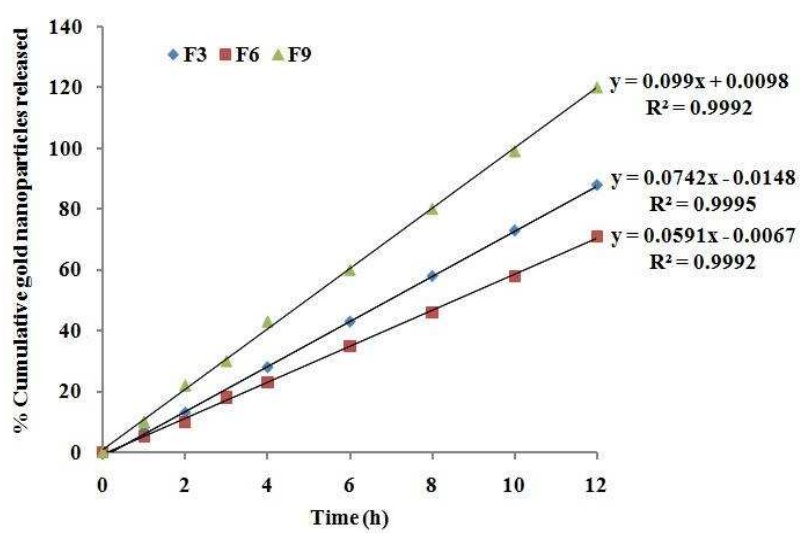

Fig. 3: Zero-order plot for the cumulative percentage of gold nanoparticles released versus time

\section{CONCLUSION}

ACV conjugated with gold nanoparticle liposomal formulations can be easily prepared with good EE\% and good drug release. The present research work could be a successful production of liposomes using lecithin and cholesterol by thin film evaporation technique. The results of the characterization and evaluation established the suitability and compatibility of the developed liposome for the use as a topical delivery system for the hydrophilic, antiviral drug, Acyclovir. Slow drug release that observed with the developed ACV-gold nanoparticles loaded liposome would be considered as advantages.

ACV encapsulation in the developed liposome had overcome the adverse effects of ACV and would offer the efficacy in herpes simplex treatment and patient compliance. Production of ACV-gold nanoparticles loaded liposome could be an effective new and commercially alternative to the commercial product.

\section{ACKNOWLEDGEMENT}

The authors would like to thank the department of pharmaceutics and industrial pharmacy, Beni-Suef University, where most of the work had been done.

\section{CONFLICT OF INTERESTS}

The authors state that no conflict of interest and have not any received payment in the preparation of this manuscript.

HEBA F. SALEM: She chose the topic on which we worked.

SAHAR M LOTAYEF: She implemented the idea in the lab and wrote the scientific paper.

SABRY M TAMAM: He reviewed the scientific paper. 


\section{REFERENCES}

1. De Clercq E, HJ Field. Antiviral prodrugs-the development of successful prodrug strategies for antiviral chemotherapy. $\mathrm{Br}$ J Pharmacol 2006;147:1-11.

2. Shah, PJ. Monitoring of Outpatient parenteral antimicrobial therapy (OPAT) and implementation of clinical pharmacy services at a community hospital infusion unit. J Pharm Pract 2015;28:462-8

3. Luengo J. Preliminary pharmacokinetic study of different preparations of acyclovir with $\beta$-cyclodextrin. J Pharm Sci Sci 2002;91:2593-8.

4. Attia IA. Influence of a niosomal formulation on the oral bioavailability of acyclovir in rabbits. AAPS PharmSciTech 2007;8:206-12.

5. Kagan L, A Hoffman. Systems for region selective drug delivery in the gastrointestinal tract: biopharmaceutical considerations. Expert Opin Drug Delivery 2008;5:681-92.

6. Godin B, E Touitou. Ethosomes: new prospects in transdermal delivery. Crit Rev Ther Drug Carrier Syst 2003;20:63-102.

7. Sivakumar P, C Nethradevi, S Renganathan. Synthesis of silver nanoparticles using lantana camara fruit extract and its effect on pathogens. Asian J Pharm Clin Res 2012;5:97-101.

8. Mezei, M, V Gulasekharam. Liposomes-a selective drug delivery system for the topical route of administration I. Lotion dosage form. Life Sci 1980;26:1473-7.

9. Touitou E. Drug delivery across the skin. Expert Opin Biol Ther 2002;2:723-33.

10. Loth H. Vehicular influence on transdermal drug penetration. Int J Pharm 1991;68:1-10.

11. Masini V. Cutaneous bioavailability in hairless rats of tretinoin in liposomes or gel. J Pharm Sci 1993;82:17-21.

12. Naveena BE, S Prakash. Biological synthesis of gold nanoparticles using marine algae Gracilaria corticata and its application as a potent antimicrobial and antioxidant agent. Asian J Pharm Clin Res 2013;6:179-82.

13. Henglein A, M Giersig. Formation of colloidal silver nanoparticles: capping action of citrate. J Phys Chem B 1999; 103:9533-9.
14. Storhoff JJ. One-pot colorimetric differentiation of polynucleotides with single base imperfections using gold nanoparticle probes. J Am Chem Soc 1998;120:1959-64.

15. Zalba S. Application of different methods to formulate PEGliposomes of oxaliplatin: evaluation in vitro and in vivo. Eur J Pharm Biopharm 2012;81:273-80.

16. Perugini P, F Pavanetto. Liposomes containing boronophenylalanine for boron neutron capture therapy. J Microencapsul 1998;15:473-83.

17. Hathout RM. Liposomes as an ocular delivery system for acetazolamide: in vitro and in vivo studies. AAPS PharmSciTech 2007;8:E1-E12.

18. Srinath P, S Vyas, PV Diwan. Preparation and pharmacodynamic evaluation of liposomes of indomethacin.Drug Dev Ind Pharm 2000;26:313-21.

19. Nagarsenker M, VY Londhe, G Nadkarni. Preparation and evaluation of liposomal formulations of tropicamide for ocular delivery. Int J Pharm 1999;190:63-71.

20. $\mathrm{Xu}, \mathrm{X}$. Effect of sodium oleate adsorption on the colloidal stability and zeta potential of detonation synthesized diamond particles in aqueous solutions. Diamond Relat Mater 2005;14:206-12.

21. Riddick TM. Control of colloid stability through zeta potential. Blood 1968;10:12-41.

22. Tabrizi A, F Ayhan, H Ayhan. Gold nanoparticle synthesis and characterisation. Hacettepe J Biol Chem 2009;37:217-26.

23. Duangjit S. Role of the charge, carbon chain length, and content of surfactant on the skin penetration of meloxicam-loaded liposomes. Int J Nanomed 2014;9:2005-17.

24. Crommelin DJ. Influence of lipid composition and ionic strength on the physical stability of liposomes. J Pharm Sci 1984;73:1559-63.

25. Hosny KM. Ciprofloxacin as an ocular liposomal hydrogel. AAPS PharmSciTech 2010;11:241-6.

\section{How to cite this article}

- Heba F Salem, Sabry M Tamam, Sahar M Lotayef Biodegradable liposomes for acyclovir-gold nanoparticles as an efficient carrier for enhanced topical delivery. Int J Pharm Pharm Sci 2017;9(8):60-64. 